\title{
Noise Induced Escape from Different Types of Chaotic Attractor
}

\author{
Igor A. Khovanov*, Vadim S. Anishchenko*, \\ Dmitri G. Luchinsky ${ }^{\dagger}$, and Peter V.E. McClintock ${ }^{\dagger}$ \\ * Department of Physics, Saratov State University, \\ Astrakhanskaya str. 83, 410026 Saratov, Russia \\ ${ }^{\dagger}$ Department of Physics, Lancaster University, Lancaster, LA1 4YB, UK
}

\begin{abstract}
Noise-induced escape from a quasi-attractor, and from the Lorenz attractor with non-fractal boundaries, are compared through measurements of optimal paths. It has been found that, for both types of attractor, there exists a most probable (optimal) escape trajectory, the prehistory of the escape being defined by the structure of the chaotic attractor. For a quasi-attractor the escape process is realized via several steps, which include transitions between low-period saddle cycles co-existing in the system phase space. The prehistory of escape from the Lorenz attractor is defined by stable and unstable manifolds of the saddle center point, and the escape itself consists of crossing the saddle cycle surrounding one of the stable point-attractors.
\end{abstract}

A major unsolved problem in the theory of fluctuations is that of noise-induced escape from a chaotic attractor [1]. Chaotic systems are widespread in nature, and the study of their dynamics in the presence of fluctuations is both of fundamental interest, and also of importance in relation to a range of applications, e.g. to stabilization of the voltage standard [2], neuron dynamics [3], and laser systems [4].

The difficulty of solving the fluctuational escape problem stems largely from the fact that the dynamics of the system during large noise-induced deviations from deterministic chaotic trajectories remains obscure. In particular, it has been unclear whether or not there exists a unique optimal path along which escape from a chaotic attractor takes place. Theoretical predictions of the character of the optimal path distribution near a chaotic attractor do not yet exist.

It has been established that fluctuational dynamics can be investigated directly through measurements of the so-called prehistory probability distribution of fluctuations [5,6], making it possible to examine situations for which the use of analytic methods still remains problematic. We have applied this technique to experimental investigations of noise-induced escape from a quasi-attractor and from the Lorenz attractor.

CP502, Stochastic and Chaotic Dynamics in the Lakes: STOCHAOS, edited by D. S. Broomhead, E. A. Luchinskaya, P.V. E. McClintock, and T. Mullin (C) 2000 American Institute of Physics 1-56396-915-7/00/\$17.00 
The essence of the prehistory technique is the idea that, when the system moves to a given remote state, it does so along an optimal path under the action of a large fluctuation [7]. The dynamical variables of the system and of the fluctuational force are recorded simultaneously, and ensemble-averages are built of the trajectories and the noise history [6]. It allows one to define both the optimal path itself and also the optimal fluctuational force inducing the escape. As a result we can evaluate the escape probability as a function of noise intensity, and we can hope to develop ways of controlling (and perhaps exploiting) large fluctuations [6,8]. This experimental approach has already facilitated studies of the symmetry of optimal paths and of the singularities in their distribution $[6,9]$.

The mathematical variant of this experimental method is the Hamiltonian approach to the asymptotic expansion of the Fokker-Plank equation to determine an optimal trajectory [10]. Use of the Hamiltonian formalism to investigate escape from a chaotic attractor requires prior answers to the questions: does a unique optimal escape path exist, and where must we take the initial state, from whence the optimal path starts? It is necessary to provide initial information about the escape process from a chaotic attractor in order to develop the theory. The experimental approach [5] allows us to define an optimal path, and it provides initial insight into the application of analytic methods.

We have chosen for investigation two systems having different types of attractor: a quasi-attractor and a quasi-hyperbolic attractor. The first system is a nonlinear oscillator forced by an external periodic signal and white noise:

$$
\begin{array}{r}
\dot{x}_{1}=x_{2}, \quad \dot{x}_{2}=-\Gamma x_{2}-\omega_{0}^{2} x_{1}-\beta x_{1}^{2}-\gamma x_{1}^{3}+h \cos \left(\omega_{f} t\right)+\xi(t), \\
\langle\xi(t)\rangle=0, \quad\langle\xi(t) \xi(0)\rangle=D \delta(t), \quad \Gamma \ll \omega_{f}, \quad \frac{9}{10}<\frac{\beta^{2}}{\gamma \omega^{2}}<4 .
\end{array}
$$

It is one that is met in many applications, and it has been studied theoretically over a wide range of parameters [11]. A chaotic attractor appears in this system via a cascade of period-doubling bifurcations, so it is a quasi-attractor [12]. We have chosen parameters $\left(\Gamma=0.05, \omega_{0}=0.5970, \beta=1, \gamma=1, \omega_{f}=0.95, h=0.13\right.$ ) for which a limit cycle and a chaotic attractor coexist.

The phase portraits of the stable limit cycle and chaotic attractor, and their basins of attraction, are shown in Fig. 1. The saddle cycle $U$ and its manifolds form a boundary between the quasi-attractor and the limit cycle. In the presence of noise there is a finite probability of noise-induced escape from the chaotic attractor. If we had a stable limit cycle instead of a quasi-attractor then, in the context of the Hamiltonian approach [10], the following escape scenario might be expected. Escape should in general occur along a unique, most probable, escape path connecting two limit cycles and crossing the unstable cycle $U$. Because the basin of attraction of the chaotic attractor is bounded by the saddle cycle $U$, the situation near it remains qualitatively the same, and therefore a unique optimal path is to be expected in this region. However, the situation arising near a chaotic attractor is unknown: the simplest scenario might be that the optimal path smears 

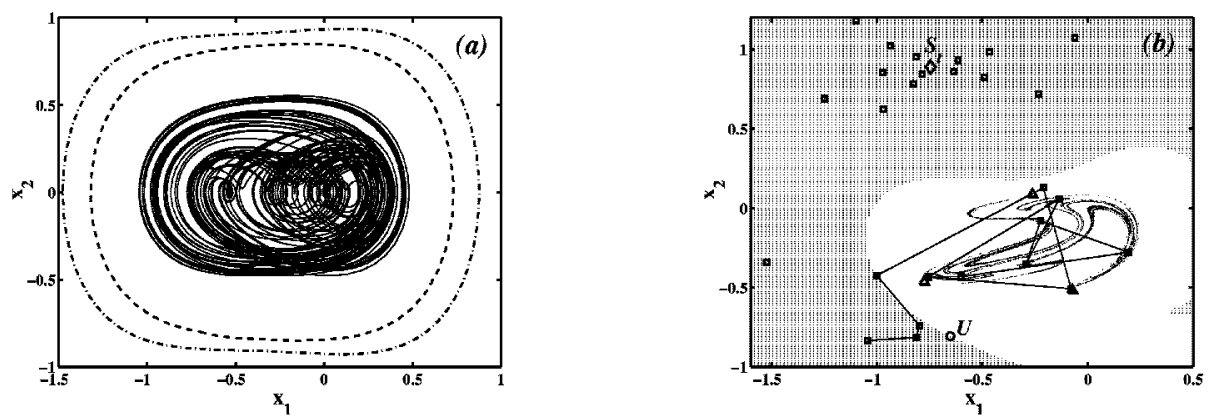

FIGURE 1. (a) The phase portrait of a chaotic attractor (solid line), the limit cycle (dot-dashed line) and the saddle cycle $U$ (dashed line). (b) The white and hatched regions correspond to the basins of attraction of the quasi-attractor (small dots) and the limit cycle $S_{t}$ (diamond) for Poincaré cross-section with $\omega_{f} t=0(\bmod 2 \pi)$. The saddle cycles $U$ and $S 3$ are shown by filled circles and triangles respectively. The intersections of the actual escape trajectory with the Poincaré cross-section are indicated by the square.

out near the boundary of the chaotic attractor, and that no unique escape path exists in this region.

Our statistical analysis of real fluctuational trajectories resolves the question immediately. Fig. 2 provides a representation of the distribution of fluctuational paths. There is a single path connecting the chaotic attractor with the stable cycle and passing through the unstable cycle $U$. This path remains single back to the boundary of the chaotic attractor, where it divides into several paths. They all merge to form an optimal path running on the saddle cycle of period $3(S 3)$, which does not belong to the quasi-attractor. Thus escape to the cycle $S 3$ may occur along several paths. We have found that these paths are defined by saddle cycles built into the structure of the quasi-attractor and that there is a unique optimal (in sense of minimal fluctuational action) path, which starts from the saddle cycle of period 5 (S5) (Fig. 2(a)).

Based on this scenario, the escape probability may be evaluated as the product of the probability of staying on the unstable cycle and the probability of subsequent transitions: from the saddle cycles embedded in the chaotic attractor to the cycle $S 3$; and from the cycle $S 3$ to the cycle $U$. In other words, the problem of escape from a quasi-attractor has been reduced to the problem of transitions between a limited number of saddle cycles. The Hamiltonian formalism [10] may therefore now be applied to investigate escape from a quasi-attractor.

We have also measured the (external) optimal fluctuational force inducing the escape. It can be related $[5,6]$ to the momentum of a Hamiltonian auxiliary system, an inference that has been verified experimentally [9]. We can expect a similar result for chaotic attractors given that, as we have shown, there exists a unique optimal escape path. As seen (Fig. 3(b)) the optimal force is composed of two distinct sections corresponding to escape to the cycles $S 3$ and $U$ respectively; it tends to 

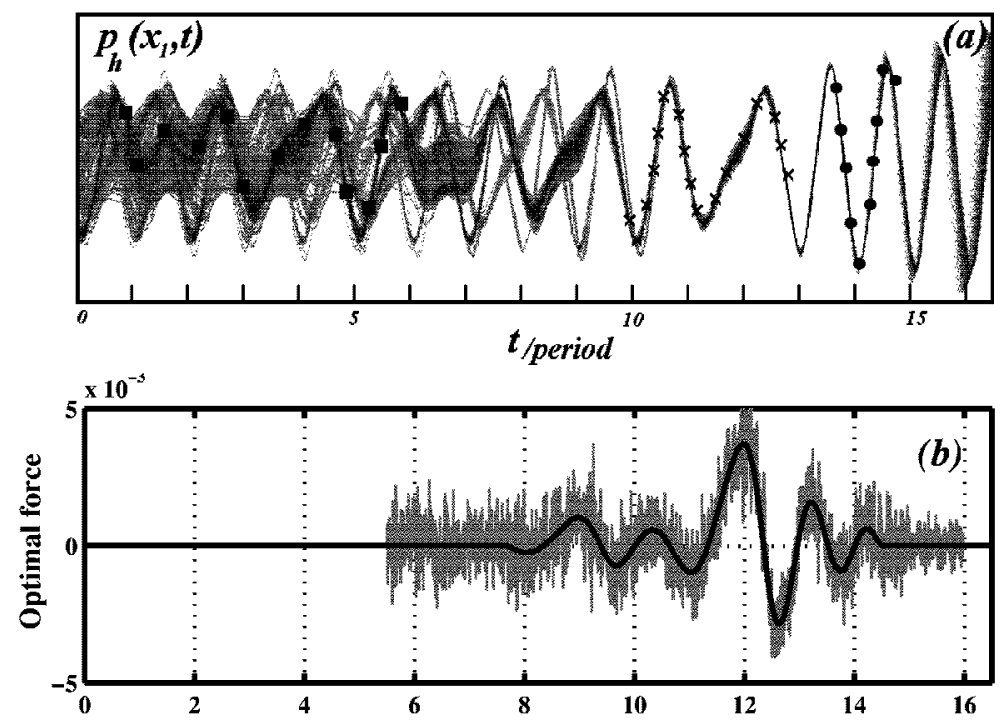

FIGURE 2. (a) The probability distribution of fluctuational paths $p_{h}\left(x_{1}, t\right)$ built up by ensemble-averaging escape trajectories. The darker colour corresponds to a larger probability. The saddle cycles $S 5, S 3$ and $U$ are shown by the symbols " $\square$ ", " $\times$ " and "•" respectively. (b) The optimal force is shown both before (grey line) and after (full curve) passage through a low-pass filter.

zero once the cycle $U$ has been attained.

We next consider fluctuational escape from the Lorenz attractor

$$
\begin{aligned}
\dot{x}_{1}=\sigma\left(x_{2}-x_{1}\right), \quad \dot{x}_{2} & =r x_{1}-x_{2}-x_{1} x_{3}, \quad \dot{x}_{3}=x_{1} x_{2}-b x_{3}+\xi(t) \\
\langle\xi(t)\rangle & =0, \quad\langle\xi(t) \xi(0)\rangle=D \delta(t),
\end{aligned}
$$

which is a quasi-hyperbolic attractor consisting of unstable sets only. This system $[13,14]$ is of interest because it describes e.g. convective fluid dynamics, as well as the single mode laser $[1,15]$. For simplicty we consider the noise source to act through the third equation only.

For $\sigma=10, b=8 / 3, r=24.08$, the system (2) has three attractors [14]: the stable points $P_{1}$ and $P_{2}$ and the Lorenz attractor (Fig. 3(a)). The stable manifolds of the saddle cycles $L_{1}$ and $L_{2}$ surround the stable points and they constitute boundaries between the chaotic and regular regimes in this region of phase space. The Lorenz attractor consists of an aggregate of integral curves going from the cycle $L_{1}$ to $L_{2}$ and back, the saddle point $O$, and its separatrices $\Gamma_{1}$ and $\Gamma_{2}$. Note that the probability of trajectories passing near the separatrices and the cycles $L_{1}$ and $L_{2}$ is practically zero for the noise-free system. Like the problem of escape from a quasi-attractor, there is no theoretical prediction for fluctuational escape from the Lorenz attractor. 

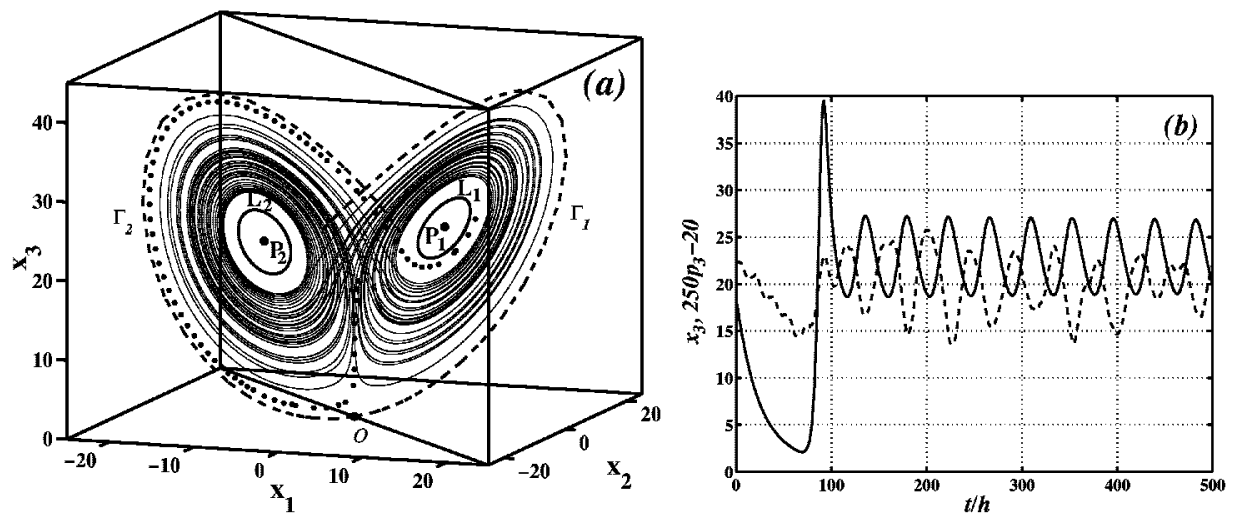

FIGURE 3. (a) The structure of phase space of the Lorenz system. One from escape trajectories is shown by points. (b) The optimal escape trajectory (solid line) and the averaged fluctuational force (dashed line) are shown.

We have investigated the problem by use of numerical simulation and the method of analysis described above in the relation to escape from a quasi-attractor. For definiteness, we consider escape to the stable point $P_{1}$. The measured optimal escape trajectory and corresponding optimal force are shown in Fig. 3.

The optimal escape trajectory starts from the stable manifold of the saddle point $O$. Under the action of a fluctuation an escape trajectory tends to point $O$ along the stable manifold. Then, without reaching the saddle point $O$, the optimal trajectory departs from it again, following a path close to the separatrix $\Gamma_{2}$, falling into the neighborhood of the saddle cycle $L_{1}$. In the absence of an external force, the trajectory has to go away from the cycle $L_{1}$ slowly untwisting. The fluctuations induce a crossing through the saddle cycle, and then the trajectory relaxes to the stable point $P_{1}$. We can therefore split the escape process to two parts: fluctuational and relaxational. Practically all of the fluctuational part belongs to the Lorenz attractor and itself consists of two stages: first, the fluctuational force throws the trajectory close to the cycle $L_{1}$; secondly, this cycle is crossed, also through the action of a fluctuation. The first stage is defined by the manifold of saddle point $O$, and the time-dependence of the fluctuational force is similar to that of the coordinate $x_{3}$ (Fig. 3(b)). During the second stage, the fluctuations have a component which oscillates in anti-phase to the coordinate $x_{3}$. The trajectory of the noise-free system departs from the cycle $L_{1}$ very slowly, so that the fluctuational force inducing the crossing through the cycle may start to act at any time during this long interval. The smoothed fluctuational force itself therefore consists of a long oscillating function.

Thus, the fluctuational part of the escape trajectory from the Lorenz attractor lies on the attractor itself. The role of the fluctuations lies in the delivery of the trajectory to a seldom-visited area in the neighborhood of the saddle cycle $L_{1}$, and 
then in inducing a crossing to $L_{1}$. To estimate the escape probability, therefore, it would suffice to build the quasi-potential of the noisy Lorenz attractor.

In summary, we have found that, for both a quasi-attractor and a quasihyperbolic attractor, a unique optimal escape path exists. We have obtained the optimal force inducing escape from both types of chaotic attractor. As a next step, we will check the possibility of optimal control of escape, using the optimal force found in numerical simulations.

The main results were obtained through the experimental definition of optimal paths, thus showing that this experimental approach is a powerful instrument for the investigation of noise-induced escape from complex attractors. Our studies of the escape process offer the possibility of a theoretical evaluation of fluctuational escape from a chaotic attractor within the framework of the well-established Hamiltonaian approach [10].

The research was supported by INTAS (grants N 96-0305, N YSF 99-3920), by the Royal Society of London, and by the Engineering \& Physical Sciences Research Council (UK).

\section{REFERENCES}

1. Graham, R., in Noise in Nonlinear Dynamical Systems vol. 1, ed. McClintock, P.V.E., and Moss, F., Cambridge University Press, 1989, p 225.

2. Kautz, R.L., Rep. Prog. Phys. 59, 935 (1996).

3. Faure, P., and Korn, H., Proc, Natl. Acad. Sci. USA 94, 6506 (1997).

4. Arimondo, E., Hennequin, D., and Glorieux, P., in Noise in Nonlinear Dynamical Systems, vol. 3, ed. McClintock, P.V.E., and Moss, F., Cambridge University Press, 1989, p 119.

5. Dykman, M.I., McClintock, P.V.E., Smelyanskiy, V.N., Stein, N.D., and Stocks, N.G., Phys. Rev. Lett. 68, 2718 (1992).

6. Luchinsky, D.G., McClintock, P.V.E., and Dykman, M.I., Rep. Prog. Phys. 61, 889 (1998).

7. Onsager, L., and Machlup, S., Phys. Rev. 91, 1505 (1953);

8. Smelyanskiy, V.N., and Dykman, M.I., Phys, Rev. E 55, 2516 (1997).

9. Luchinsky, D.G., and McClintock, P.V.E., Nature 389, 463 (1997); Luchinsky, D.G., Maier, R.S., Mannella, R., McClintock, P.V.E., Stein, D.L., Phys. Rev. Lett. 79, 3117 (1997); Luchinsky, D.G., J. Phys. A 30, L577 (1997).

10. Freidlin, M.I., and Wentzel, A.D., Random Perturbations in Dynamical Systems, Springer, New-York, 1984.

11. Mannella, R., Soskin, S.M., and McClintock, P.V.E., Int. J. of Bifurcation and Chaos 8, 701 (1998).

12. Afraimovich, V.S., in Nonlinear and turbulent process in physics, Gordon and Breach, New York, 1984, pp 1133-1138.

13. Lorenz, E.N. J. Atmos. Sci. 20, 130 (1963).

14. Sparrow, C., Appl. Math. Sci. 41, 269 (1982).

15. Haken, H., Phys. Lett. A 53, 77 (1975). 
Copyright $\odot 2003$ EBSCO Publishing 
Copyright of AIP Conference Proceedings is the property of American Institute of Physics and its content may not be copied or emailed to multiple sites or posted to a listserv without the copyright holder's express written permission. However, users may print, download, or email articles for individual use. 